\title{
Earplugs in the ICU: To sleep, to dream
}

\author{
Edward Litton ${ }^{1,2^{*}}$, Rosalind Elliott ${ }^{3}$ and Kelly Thompson ${ }^{4}$ \\ See related research by Demoule et al., https://ccforum.biomedcentral.com/articles/10.1186/s13054-017-1865-0
}

In the November issue of Critical Care, Demoule et al. [1] describe a randomized controlled trial (RCT) undertaken in unsedated patients admitted to the intensive care unit (ICU), assessing the addition of earplugs and eye masks to routine care alone, on polysomnographic measures of sleep quality. The study provides novel mechanistic data supporting the efficacy of earplugs to improve sleep in critically ill patients, but also highlights the need for deeper methodological considerations.

First, the per protocol analysis introduces the risk of selection bias. This would not be decreased by greater participant numbers. Compliant participants in the subgroup that wore earplugs for the entire study period may also have been less anxious and less prone to sleep disturbance. They may also have found earplug insertion more comfortable and effective. Optimizing earplug choice and insertion training could mitigate this effect by improving compliance, as both can substantially increase tolerability and sound abatement $[2,3]$. In our recent pilot RCT of earplugs in patients admitted to the ICU, ease of insertion and participant-reported earplug comfort were rated highly and resulted in an intention- to-treat analysis in which earplug use occurred in $78 \%$ of the overnight intervention hours [4].

Second, definitive evaluation of whether earplugs improve patient-centered outcomes requires maximizing exposure to the intervention in the group of patients most likely to benefit. Demoule et al. [1] excluded participants receiving sedation, although these patients constitute a large proportion of the case-mix of most ICUs, are often at high risk for adverse outcomes, and may still benefit from decreased noise-associated arousals. Furthermore, polysomnography results were reported from only a single night and whether any effect on prolonged awakenings is sustained over the subsequent ICU stay is uncertain. An inadequate intervention period may also explain why participant-rated sleep quality was not improved by the intervention.

Whilst this study adds to the growing body of evidence to support earplugs as a plausible candidate intervention to reduce sleep disruption in patients admitted to the ICU, high quality evidence demonstrating improved patient-centered outcomes is required before widespread adoption of this intervention is warranted.

\section{Authors' response}

\section{Serge Carreira, Thomas Similowski and Alexandre Demoule}

We thank Dr Litton and colleagues for reading our paper.

First, we agree that per-protocol analysis introduces the risk of selection bias. However, blind randomization does not totally prevent from bias in patient selection because it does not identify patient preferences or reactions to and compliance with the treatment assignment [5]. In our study, $30 \%$ of the patients in the intervention group abandoned the intervention. The challenge is therefore to identify a priori and reliably the patients who would tolerate the device, which is a form of precision medicine [6]. In their pilot study, Litton et al. [4]

\footnotetext{
*Correspondence: ed_litton@hotmail.com; ed.litton@health.wa.gov.au

${ }^{1}$ Intensive Care Unit, Fiona Stanley Hospital, Perth, Western Australia, Australia

${ }^{2}$ St John of God Hospital, Subiaco, Perth, Western Australia, Australia

Full list of author information is available at the end of the article
}

showed that pre-inclusion training was an attractive option. However, this strategy cannot be applied to every critical care patient (see below).

Second, Litton et al. pointed out rightly that we did not include patients receiving sedation. We made this choice since sedation deeply alters sleep architecture [7]. Detecting a positive impact of earplugs and eye mask on such recordings would be difficult and implies a clear definition of polysomnographic criteria of improvement. In addition, the fact that a substantial proportion of our patients removed their earplugs and eye mask because of claustrophobia or discomfort questions the inclusion of sedated patients in such a trial. Because of sedation, those patients could not consent themselves and would subsequently be exposed to discomfort and claustrophobia 
without their approval and without the possibility of complaining, inasmuch as the intubation probe does not allow them to speak. Obviously, pre-inclusion training as suggested by Linton et al. could not be performed in patients receiving too deep sedation.

Finally, Litton et al. suggest that repeating polysomnography recordings would have provided more information on the impact of earplugs and eye masks on sleep. Although we cannot rule out this seducing hypothesis, we would like to point out that our experience based on our study suggests that polysomnography is not so well accepted by patients and that not all of them would accept it repeatedly. Actually, in the first version of our protocol, polysomnography was planned on the two first nights following inclusion. A preliminary study showed that many patients eventually refused the second polysomnography, which forced us to ultimately modify the study protocol.

In conclusion, we believe that the limitations of our study are related to the major issues of sleep research in ICU patients: first, better patient selection because one treatment does not fit all; and second, the development of less invasive tools to reliably assess sleep architecture.

\section{Funding}

Not applicable.

Availability of data and materials

Not applicable.

\section{Authors' contributions}

All authors contributed equally to the submission. All authors read and approved the final manuscript.

Ethics approval and consent to participate

Not applicable.

\section{Consent for publication}

Not applicable.

\section{Competing interests}

The authors declare that they have no competing interests.

\section{Publisher's Note}

Springer Nature remains neutral with regard to jurisdictional claims in published maps and institutional affiliations.

\section{Author details}

${ }^{1}$ Intensive Care Unit, Fiona Stanley Hospital, Perth, Western Australia, Australia. ${ }^{2}$ St John of God Hospital, Subiaco, Perth, Western Australia, Australia. ${ }^{3}$ Intensive Care Unit, Royal North Shore Hospital, Sydney, NSW, Australia. ${ }^{4}$ Critical Care and Trauma Division, The George Institute for Global Health, Sydney, NSW, Australia.

Received: 28 November 2017 Accepted: 12 January 2018

Published online: 22 February 2018

\section{References}

1. Demoule A, Carreira S, Lavault S, Pallanca O, Morawiec E, Mayaux J, Arnulf I, Similowski T. Impact of earplugs and eye mask on sleep in critically ill patients: a prospective randomized study. Crit Care. 2017;21(1):284.
2. Chisholm EJ, Kuchai R, McPartlin D. An objective evaluation of the waterproofing qualities, ease of insertion and comfort of commonly available earplugs. Clin Otolaryngol Allied Sci. 2004;29(2):128-32.

3. Salmani Nodoushan M, Mehrparvar AH, Torab Jahromi M, Safaei S, Mollasadeghi A. Training in using earplugs or using earplugs with a higher than necessary noise reduction rating? A randomized clinical trial. Int J Occup Environ Med. 2014;5(4):187-93.

4. Litton E, Elliott R, Ferrier J, Webb SAR. Quality sleep using earplugs in the intensive care unit: the QUIET pilot randomised controlled trial. Crit Care Resusc. 2017;19(2):128-33.

5. Sackett $\mathrm{DL}$, Wennberg JE. Choosing the best research design for each question. BMJ. 1997;315:1636

6. Seymour CW, Gomez H, Chang CH, Clermont G, Kellum JA, Kennedy J, Yende S, Angus DC. Precision medicine for all? Challenges and opportunities for a precision medicine approach to critical illness. Crit Care. 2017;21:257

7. McEntire DM, Kirkpatrick DR, Kerfeld MJ, Hambsch ZJ, Reisbig MD, Agrawal DK, Youngblood CF. Effect of sedative-hypnotics, anesthetics and analgesics on sleep architecture in obstructive sleep apnea. Expert Rev Clin Pharmacol. 2014;7:787-806. 